\title{
Utility of D2-40, a novel mesothelial marker, in the diagnosis of malignant mesothelioma
}

\author{
Albert Y Chu, Leslie A Litzky, Theresa L Pasha, Geza Acs and Paul J Zhang \\ Department of Pathology and Laboratory Medicine, Hospital of the University of Pennsylvania, Philadelphia, \\ $P A, U S A$.
}

\begin{abstract}
Although immunohistochemistry has proven to be valuable in the differentiation of epithelioid mesothelioma from pulmonary or metastatic adenocarcinoma, no single antibody has demonstrated absolute sensitivity or specificity in making this distinction. Using immunohistochemical analysis with D2-40, a recently available monoclonal antibody that has been used as a lymphatic endothelial marker, we examined 53 cases of mesothelioma, 28 cases of reactive pleura, 30 cases of pulmonary adenocarcinoma, 35 cases of renal cell carcinoma, 26 cases of ovarian serous carcinoma, 16 cases of invasive breast carcinoma, 11 cases of prostatic adenocarcinoma, and seven cases of urothelial carcinoma. In addition, immunohistochemistry using calretinin, cytokeratin 5/6, and WT1 was performed on all cases of mesothelioma, pulmonary adenocarcinoma, ovarian serous carcinoma, and renal cell carcinoma. Predominantly, membranous D2-40 immunoreactivity was present in 51 of $53(96 \%)$ mesotheliomas, 27 of $28(96 \%)$ cases of reactive pleura, and 17 of 26 (65\%) ovarian serous carcinomas; membranous staining was not seen in any other tumors examined. Compared to other immunohistochemical markers of mesothelioma, D2-40 was as sensitive as calretinin and more sensitive than cytokeratin 5/6 and WT1. We conclude that D2-40 immunoreactivity is sensitive for cells of mesothelial origin, and may be useful in the differential diagnosis of epithelioid malignant mesothelioma vs adenocarcinoma. Modern Pathology (2005) 18, 105-110, advance online publication, 24 September 2004; doi:10.1038/modpathol.3800259
\end{abstract}

Keywords: mesothelioma; D2-40; immunohistochemistry; calretinin; cytokeratin 5/6; WT1

Malignant mesothelioma, with its wide differential diagnosis and prognostic implications, often presents a diagnostic issue in surgical pathology. In addition to evaluation by conventional light microscopy, multiple ancillary techniques including electron microscopy and immunohistochemistry have been employed to increase the accuracy of diagnosis. While the development of new immunohistochemical markers has improved our diagnostic ability, no single antibody has demonstrated absolute sensitivity or specificity for malignant mesothelioma. ${ }^{1-5}$ As a result, pathology laboratories typically employ a panel of immunohistochemical markers known to be either positive or negative for mesothelioma in the differential diagnosis of the disease. ${ }^{5-7}$

D2-40 is a recently developed, commercially available monoclonal antibody directed against

Correspondence: Dr PJ Zhang, MD, Department of Pathology and Laboratory Medicine, Hospital of the University of Pennsylvania, 6 Founders Pavilion, 3400 Spruce Street, Philadelphia, PA 19104, USA.

E-mail: pjz@mail.med.upenn.edu

Received 28 May 2004; revised and accepted 26 July 2004; published online 24 September 2004
M2A antigen, an $M_{\mathrm{r}} 40000$ surface sialoglycoprotein originally detected in association with germ cell neoplasia and fetal testicular gonocytes. ${ }^{8}$ Since D240 has also demonstrated selective immunoreactivity for lymphatic endothelium, ${ }^{9}$ its proposed clinical uses include the demonstration of lymphatic invasion by primary tumors and its use as a marker of certain vascular lesions. ${ }^{9-12}$ In addition to this previously described selective immunoreactivity for germ cells and lymphatic endothelium, we recently observed D2-40 staining in cells of mesothelial origin. To evaluate the potential utility of this antibody in making a diagnosis of malignant mesothelioma, we performed immunohistochemical analysis using D2-40 on cases of malignant mesothelioma and its potential histologic mimics, including reactive pleural fibrosis, pulmonary adenocarcinoma, and selected carcinomas potentially metastatic to the lung. To directly compare the performance of D2-40 in diagnosing malignant mesothelioma with currently used 'positive' immunohistochemical markers, we also performed immunohistochemistry using a panel of calretinin, cytokeratin $5 / 6$, and WT1 on all cases of mesothelioma and a subset of carcinomas including those of pulmonary, renal, and ovarian origin. 


\section{Materials and methods}

\section{Clinical Specimens}

Cases were retrieved from the files of the Department of Pathology and Laboratory Medicine, Division of Anatomic Pathology, at the Hospital of the University of Pennsylvania. The study group consisted of 53 cases of pleural malignant mesothelioma (33 epithelioid, 16 biphasic, and four sarcomatoid), 31 cases of reactive pleura, 30 cases of pulmonary adenocarcinoma, 35 cases of renal cell carcinoma (16 conventional, 13 papillary, and six chromophobe), 26 cases of ovarian serous carcinoma, 16 cases of invasive breast carcinoma, 11 cases of prostatic adenocarcinoma, and seven cases of urothelial carcinoma. The diagnosis of mesothelioma was based upon the clinical presentation, histologic features and immunohistochemical evaluation and/ or ultrastructural analysis according to the current criteria for the diagnosis of mesothelioma. ${ }^{13}$ Immunostaining for cytokeratin, CEA, calretinin, B72.3, and/or CD15 were commonly used at the time of the diagnosis. The original histologic material including the immunohistochemical staining of all mesotheliomas were reviewed by the authors (AYC, LAL, and PJZ) to confirm the diagnosis. One representative paraffin block from each case was used for the study.

\section{Immunohistochemistry}

Immunohistochemistry using D2-40 was performed in all cases; in addition, calretinin, WT1, and cytokeratin 5/6 immunostaining was performed in cases of mesothelioma, pulmonary adenocarcinoma, renal cell carcinoma, and ovarian serous carcinoma. One case of epithelioid mesothelioma was not available for cytokeratin 5/6 staining.

For this analysis, formalin-fixed paraffin-embedded tissue was sectioned at $5 \mu \mathrm{m}$ on Fisherbrand Superfrost/Plus slides (Fisher Scientific, Pittsburgh, PA, USA). Antibodies to M2A antigen (clone D2-40, 1:25, Signet Laboratories, Dedham, MA, USA), WT1 (clone 6F-H2, 1:400, DakoCytomation, Carpinteria, CA, USA), calretinin (rabbit polyclonal, 1:50, Zymed Laboratories, South San Francisco, CA, USA), and cytokeratin 5/6 (clone D5/16 B4, 1:25, DakoCytomation) were immunostained using the Envision + HRP detection system via the DakoCytomation Autostainer. Prior to immunostaining, pretreatment was performed for each antibody as follows: D2-40 was boiled in $1 \times$ EDTA pH 8.0 (Lab Vision Corporation, Fremont, CA, USA) at $95-100^{\circ} \mathrm{C}$ for $20 \mathrm{~min}$, WT1 was steamed in $1 \times$ citrate buffer $\mathrm{pH} \quad 6.0$ (Lab Vision) at $95-100^{\circ} \mathrm{C}$ for $20 \mathrm{~min}$, calretinin was microwaved in $1 \times$ citrate buffer $\mathrm{pH}$ 6.0 (Lab Vision) for $4 \mathrm{~min}$ at $70 \%$ power $(1200 \mathrm{~W}$ microwave oven) $\times 2$, and cytokeratin $5 / 6$ was boiled in $1 \times$ EDTA pH 8.0 (Lab Vision) at 95$100^{\circ} \mathrm{C}$ for $20 \mathrm{~min}$. After pretreatment, all specimens were cooled for $20 \mathrm{~min}$ in their respective retrieval buffers. Slides were then transferred to fresh wash buffer for $10 \mathrm{~min}$ and then loaded on the instrument for staining.

The intensity (on a scale of $0-4+$ ), percentage, and pattern of immunostaining were evaluated and recorded semiquantitatively by light microscopy.

\section{Results}

Results of D2-40 immunohistochemistry are summarized in Table 1; staining characteristics of D2-40positive samples are summarized in Table 2 . Membranous and/or luminal immunoreactivity was seen in tissues of mesothelial origin, including benign mesothelium $(27 / 28,96 \%)$ and epithelioid mesothelioma (33/33, 100\%) (Figures 1 and 2). In some cases with very intensive membrane staining, a less-intensive cytoplasmic staining was also observed. Membranous D2-40 immunoreactivity was also seen in most areas of epithelioid differentiation of biphasic mesothelioma (15/16, 94\%). Staining in areas of sarcomatoid differentiation (either biphasic mesothelioma or pure sarcomatoid subtype) was less frequent (10 of 16 biphasic, three of four sarcomatoid), less intense, and with a cytoplasmic pattern (Figure 3). In addition, a high level of cytoplasmic immunoreactivity was present in the entrapped reactive pleural tissue and desmoplastic tissue surrounding infiltrating tumor cells in sarcomatoid cases. A membranous pattern of D2-40 staining was also seen in 17 of 26 (65\%) ovarian serous carcinomas (Figure 4a); however, the staining was more focally distributed and less intense compared to cases of reactive pleura and epithelioid mesothelioma. D2-40 immunoreactivity was seen in only two of $30(7 \%)$ cases of pulmonary adenocarcinomas, with only focal weak cytoplasmic staining seen in the remaining two cases. Focal weak cytoplasmic staining was also seen in three of 16 $(19 \%)$ of invasive breast carcinomas, and five of 13 $(38.5 \%)$ papillary renal cell carcinomas (Figure $4 \mathrm{~b})$.

Table 1 D2-40 staining, all samples

\begin{tabular}{lrc}
\hline Tissue & $\mathrm{n}$ & D2-40 positive (\%) \\
\hline Malignant mesothelioma, all subtypes & 53 & $51(96)$ \\
Epithelioid & 33 & $33(100)$ \\
Biphasic & 16 & $15(94)$ \\
Sarcomatoid & 4 & $3(75)$ \\
Reactive pleura & 28 & $27(96)$ \\
Ovarian serous carcinoma & 26 & $17(65)$ \\
Renal cell carcinoma, all subtypes & 34 & $5(15)$ \\
Papillary & 13 & $5(39)$ \\
Conventional & 16 & $0(0)$ \\
Chromophobe & 5 & $0(0)$ \\
Breast carcinoma, invasive & 16 & $3(19)$ \\
Pulmonary adenocarcinoma & 31 & $2(7)$ \\
Prostatic adenocarcinoma & 11 & $0(0)$ \\
Urothelial carcinoma & 7 & $0(0)$ \\
\end{tabular}


Table 2 D2-40-positive samples, staining characteristics

\begin{tabular}{|c|c|c|c|c|}
\hline Tissue & $\mathrm{n}$ & $\%$ cells staining, mean & Staining intensity, median & Predominant staining pattern \\
\hline \multicolumn{5}{|l|}{ Malignant mesothelioma } \\
\hline Epithelioid & 33 & 92 & $4+$ & Membranous \\
\hline Biphasic & 15 & $90^{\mathrm{a}}, 26^{\mathrm{b}}$ & $3+^{\mathrm{a}}, 1+^{\mathrm{b}}$ & Membranous $^{\mathrm{a}}$, cytoplasmic ${ }^{\mathrm{b}}$ \\
\hline Sarcomatoid & 3 & 52 & $1+$ & Cytoplasmic \\
\hline Reactive pleura & 27 & 71 & $4+$ & Membranous \\
\hline Ovarian serous carcinoma & 17 & 33 & $2 / 3+$ & Membranous \\
\hline Renal cell carcinoma, papillary & 5 & 43 & $1 / 2+$ & Cytoplasmic \\
\hline Breast carcinoma, invasive & 3 & 13 & $2+$ & Cytoplasmic \\
\hline Pulmonary adenocarcinoma & 2 & 13 & $2+$ & Cytoplasmic \\
\hline
\end{tabular}

${ }^{\mathrm{a}}$ Epithelioid areas.

${ }^{\mathrm{b}}$ Sarcomatoid areas.

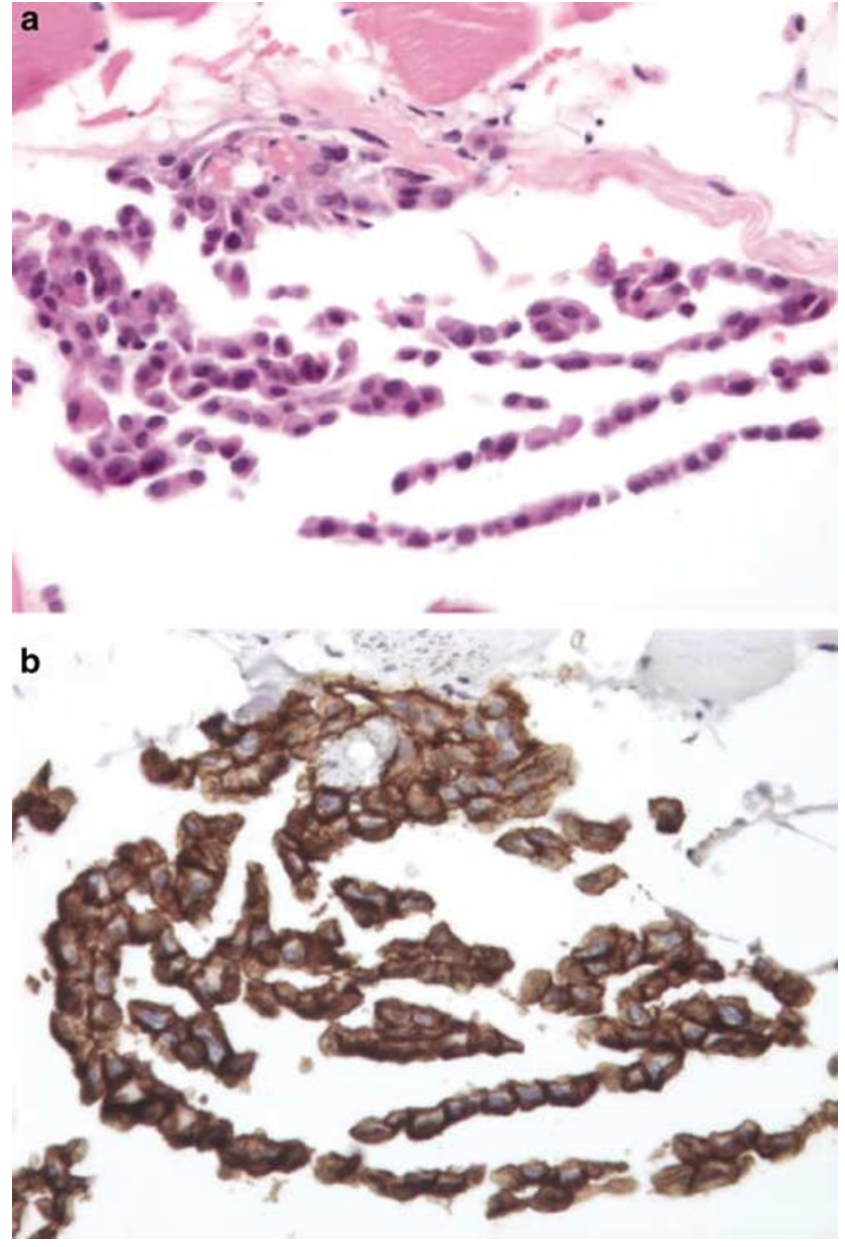

Figure 1 Benign mesothelium. High-power view showing benign strips of pleural mesothelial cells. (a) Hematoxylin \& eosin. (b) D2-40 immunostain showing intense membranous reactivity.

All cases of conventional renal cell carcinoma, chromophobe renal cell carcinoma, urothelial carcinoma, and prostatic adenocarcinoma were negative for D2-40 immunoreactivity. In all cases, D2-40 highlighted the endothelium of lymphatic vessels when present in the tissue section in a thin smooth linear staining pattern, which was very distinct from that seen in mesothelioma.

The results of immunohistochemistry using calretinin, cytokeratin $5 / 6$, and WT1 in cases of mesothelioma, pulmonary adenocarcinoma, ovarian serous carcinoma, and renal cell carcinoma are summarized in Table 3. Nuclear and cytoplasmic calretinin immunoreactivity was present in all cases of epithelioid mesothelioma (33/33) and epithelioid areas of differentiation in cases of biphasic mesothelioma (16/16). Seven of $31(23 \%)$ cases of pulmonary adenocarcinoma focally reacted for calretinin; these cases tended to be more poorly differentiated histologically. Cytoplasmic cytokeratin 5/6 staining was present in most cases of epithelioid (29/32, $91 \%)$ and biphasic $(13 / 16,81 \%)$ mesothelioma, but also in 12 of $31(39 \%)$ of pulmonary adenocarcinomas, 13 of $35(37 \%)$ renal cell carcinomas, and nine of $26(35 \%)$ ovarian serous carcinomas; cytokeratin $5 / 6$ staining in these nonmesothelioma tumors tended to be focal and weak. WT1 was the least sensitive marker of mesothelioma tested, demonstrating nuclear staining in 29 of $53(55 \%)$ cases of all mesotheliomas and 19 of $33(57 \%)$ cases of the epithelioid subtype.

\section{Discussion}

The increasing availability of 'positive' immunohistochemical markers such as calretinin, cytokeratin $5 / 6$, and WT1 has greatly aided in the diagnosis of malignant mesothelioma. The utility of these antibodies is limited, however, by their lack of absolute sensitivity or specificity, particularly in cases of mesothelioma that lack an epithelioid growth pattern. As a result, it is common practice for pathologists to employ a panel of both positive and negative markers of mesothelioma, with the final diagnosis relying on a probabilistic approach that takes into account the overall immunophenotype. Until a single ancillary technique that reliably demonstrates both high sensitivity and specificity for malignant mesothelioma is developed, 

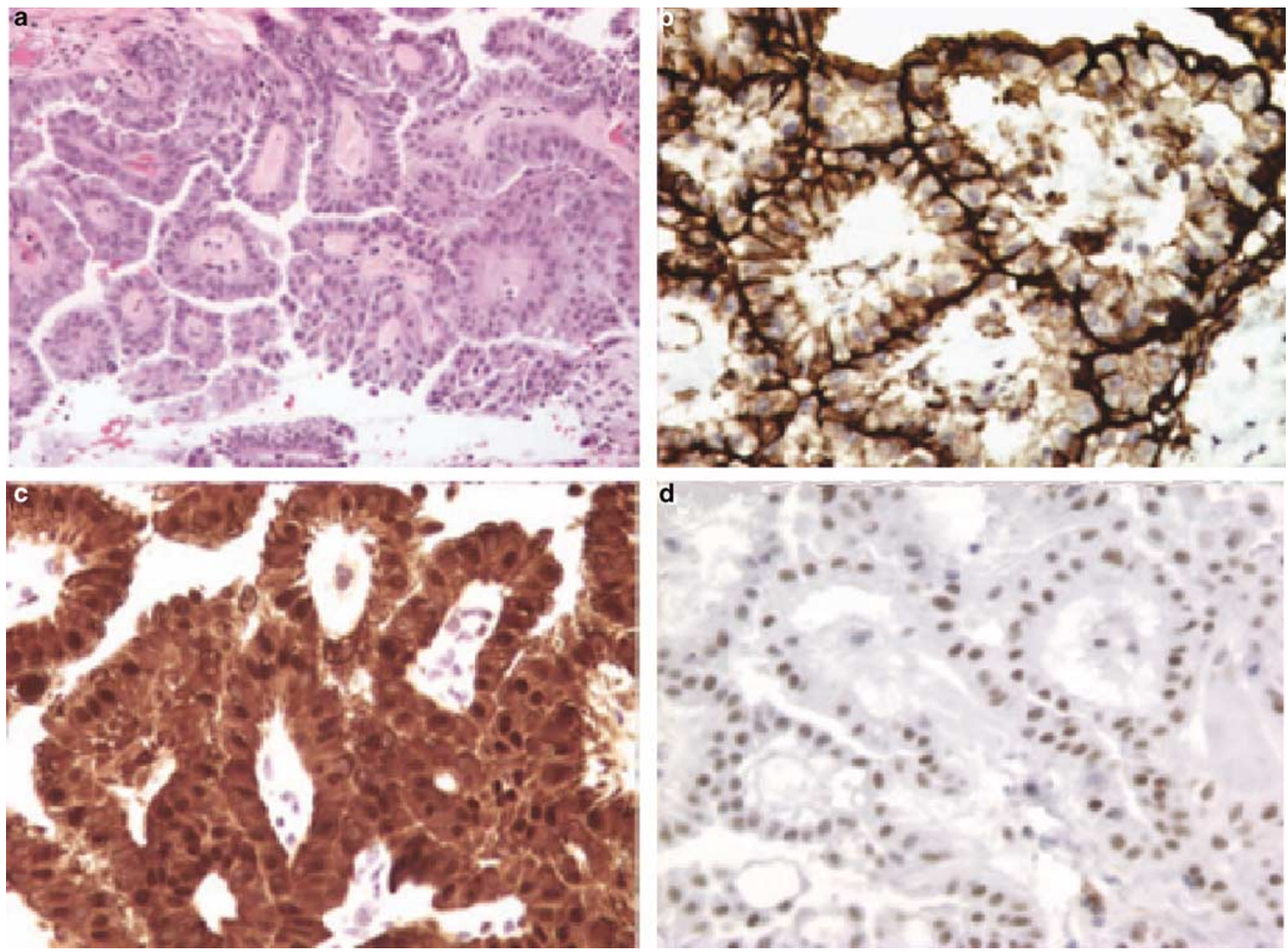

Figure 2 Malignant mesothelioma, epithelioid subtype. (a) Intermediate-power view showing epithelioid tumor cells arranged in a papillary architecture. (b) High power of D2-40 immunostain illustrating membranous pattern of immunoreactivity with highlighting of cell borders. This case was also positive for calretinin (c) and WT1 (d), and negative for cytokeratin 5/6. Note the distinct difference in staining pattern among D2-40 (membrane), calretinin (cytoplasmic), and WT-1 (nuclear).

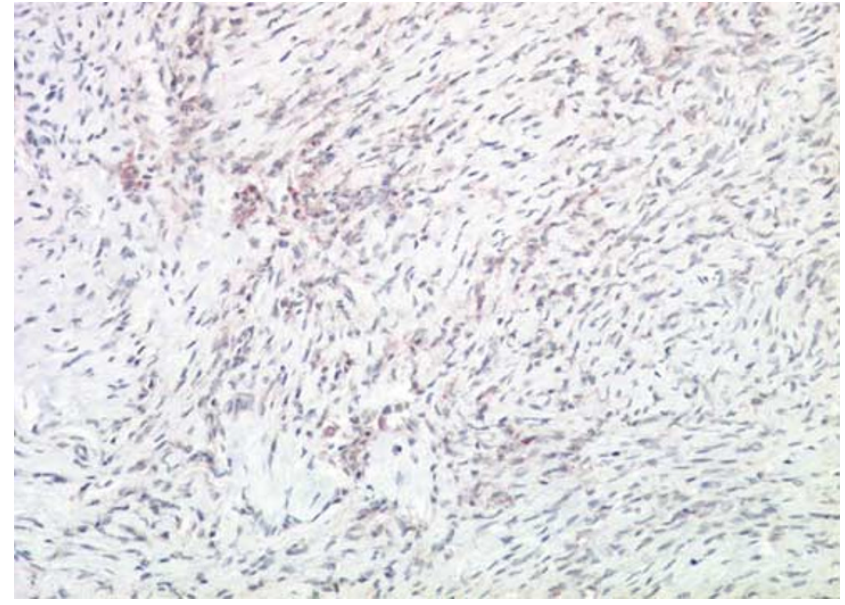

Figure 3 Malignant mesothelioma, sarcomatoid type. D2-40 immunostain showing focal weak cytoplasmic reactivity.

evaluation of immunohistochemical markers for use in a diagnostic panel is likely to continue.

In this study, we have shown that D2-40, a monoclonal antibody directed against oncofetal antigen M2A and a selective marker of germ cells and lymphatic endothelium, is also a novel and sensitive marker for cells of mesothelial origin, and its sensitivity for mesothelioma, particularly in areas of epithelioid differentiation, is as good or superior to other currently used positive markers of mesothelioma. In differentiating epithelioid malignant mesothelioma from pulmonary adenocarcinoma, D2-40 also demonstrates relatively high specificity, particularly when the characteristic membranous staining pattern of mesothelial-derived cells is taken into account. Although a small number of adenocarcinomas of nonserous origin demonstrated focal cytoplasmic immunoreactivity, none demonstrated membranous D2-40 reactivity. Thus, recognition of this membranous staining pattern may also be particularly useful in distinguishing malignant mesothelioma from various adenocarcinomas such as breast carcinoma and especially renal cell carcinoma for which there are no currently available highly sensitive and specific markers. ${ }^{14}$ The additional presence of membranous D2-40 immunoreactivity in a significant proportion of ovarian serous 
carcinomas is comparable to previously described WT1 nuclear reactivity in both malignant mesotheliomas and ovarian serous carcinomas, likely reflect-

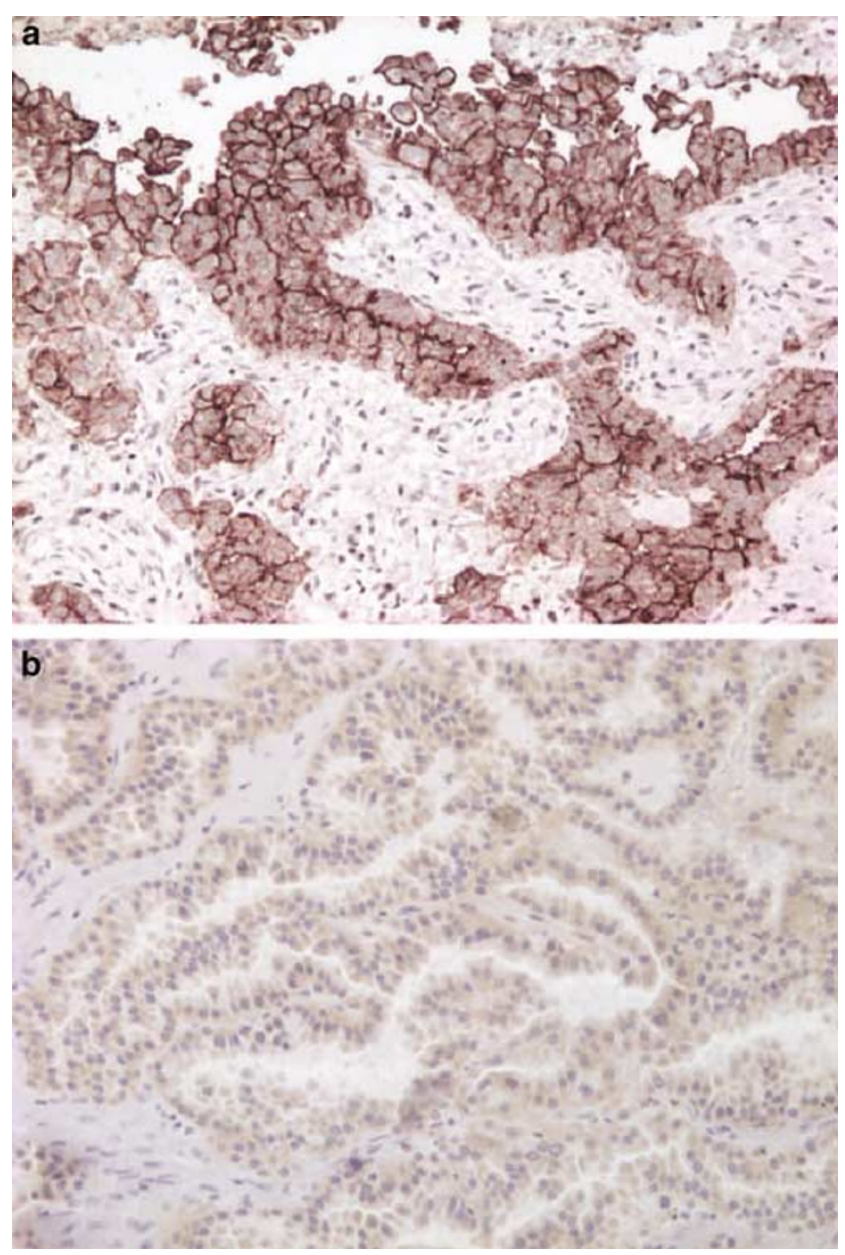

Figure 4 D2-40 immunoreactivity in ovarian serous carcinoma and papillary renal cell carcinoma. (a) Ovarian serous carcinoma, showing membranous immunoreactivity similar to that seen in epithelioid mesothelioma. (b) Papillary renal cell carcinoma, exhibiting diffuse but weak D2-40 immunostaining in a cytoplasmic pattern. ing the close histogenetic relationship between mesothelium and serosal lining cells of Mullerian origin. $^{15}$

In a recent study, and review of currently available immunohistochemical markers, Ordóñez ${ }^{7}$ reported that calretinin, cytokeratin $5 / 6$, and WT1 demonstrated the highest sensitivity for epithelioid mesothelioma and recommended the use of these antibodies as positive markers in an immunohistochemical panel for making this diagnosis. Within the present study, we found that the sensitivity of D2-40 was equal to that of calretinin, slightly higher than that of cytokeratin 5/6, and markedly better than that of WT1. In the differentiation of mesothelioma from pulmonary adenocarcinoma, D2-40 demonstrated specificity equal to that of WT1 and superior to that of calretinin and cytokeratin 5/6.

The frequency of WT1 staining in epithelioid mesothelioma in the current study is significantly lower than that observed in most previous studies. ${ }^{7}$ While possible explanations for this include the use of a different antibody (monoclonal vs polyclonal) and variation in antibody dilution and antigen retrieval methods compared to other studies, the observation that the degree of WT1 staining in ovarian serous carcinomas in our study is comparable to those previously reported in. ${ }^{16-20}$ This suggests that the lack of sensitivity of WT1 in our laboratory for mesothelioma is due to an as-yetunidentified variable external to our immunohistochemical methodology (eg factors related to tissue fixation and processing) rather than a problem with the optimization of the use of the WT1 antibody itself. From a practical standpoint, this exemplifies the phenomenon of interlaboratory variability and demonstrates the utility of developing multiple potentially interchangeable and redundant markers, particularly when a panel approach is used for diagnosis.

As with other currently used immunohistochemical markers of malignant mesothelioma, ${ }^{21,22}$ D2-40 is limited by its performance in areas of sarcomatoid differentiation. In addition to the reduced frequency

Table 3 D2-40, calretinin, WT1, and cytokeratin 5/6 staining characteristics in selected tumors

\begin{tabular}{|c|c|c|c|c|c|}
\hline & $\mathrm{n}$ & D2-40 positive (\%) & Calretinin positive (\%) & WT1 positive (\%) & CK $5 / 6$ positive (\%) \\
\hline Mesothelioma, all subtypes & 53 & $51(96)$ & $51(96)$ & $29(55)$ & $43(83)^{\mathrm{a}}$ \\
\hline Epithelioid & 33 & $33(100)$ & $33(100)$ & $19(58)$ & $29(91)^{\mathrm{a}}$ \\
\hline Biphasic & 16 & $15(94)$ & $16(100)$ & $10(63)$ & $13(81)$ \\
\hline Sarcomatoid & 4 & $3(75)$ & $2(50)$ & $0(0)$ & $1(25)$ \\
\hline Pulmonary adenocarcinoma & 31 & $2(7)$ & $7(23)$ & $2(7)$ & $12(39)$ \\
\hline Ovarian serous carcinoma & 26 & $17(65)^{\mathrm{b}}$ & $8(31)$ & $23(89)$ & $9(35)$ \\
\hline Renal cell carcinoma, all subtypes & 35 & $5(14)$ & $4(11)$ & $1(3)$ & $13(37)$ \\
\hline Conventional & 16 & $0(0)$ & $2(13)$ & $0(0)$ & $5(31)$ \\
\hline Papillary & 13 & $5(39)^{\mathrm{c}}$ & $1(8)$ & $1(8)$ & $7(54)$ \\
\hline Chromophobe & 6 & $0(0)$ & $1(17)$ & $1(17)$ & $1(17)$ \\
\hline
\end{tabular}

${ }^{\mathrm{a}}$ One case of epithelioid mesothelioma was unavailable for cytokeratin 5/6 staining.

${ }^{\mathrm{b}}$ All D2-40-positive ovarian serous carcinomas demonstrated a membranous staining pattern.

${ }^{\mathrm{c}}$ All D2-40-positive papillary renal cell carcinomas demonstrated a cytoplasmic staining pattern. 
of positively staining tumor cells, the interpretation of D2-40 staining in areas of sarcomatoid growth is complicated by the loss of the characteristic membranous staining pattern seen in epithelioid mesotheliomas and the high level of background staining present in both benign reactive pleural tissue and desmoplastic tissue surrounding infiltrating tumor cells. As a result of these factors, it is likely that the clinical utility of D2-40 will be mainly limited to malignant mesothelioma of the epithelioid subtype.

In summary, we report that monoclonal D2-40, previously described as a selective marker of germ cells and lymphatic endothelium, is also a novel marker of cells with a mesothelial phenotype, and may be useful in the differential diagnosis of epithelioid malignant mesothelioma vs adenocarcinoma. The sensitivity and specificity of this antibody is comparable or superior to currently available positive markers of epithelioid malignant mesothelioma and its inclusion in an immunohistochemical panel used to make this diagnosis may be warranted.

\section{References}

1 Amin KM, Litzky LA, Smythe WR, et al. Wilms' tumor 1 susceptibility (WT1) gene products are selectively expressed in malignant mesothelioma. Am J Pathol 1995;146:344-356.

2 Clover J, Oates J, Edwards C. Anti-cytokeratin 5/6: a positive marker for epithelioid mesothelioma. Histopathology 1997;31:140-143.

3 Doglioni C, Tos AP, Laurino L, et al. Calretinin: a novel immunocytochemical marker for mesothelioma. Am J Surg Pathol 1996;20:1037-1046.

4 Miettinen M, Sarlomo-Rikala M. Expression of calretinin, thrombomodulin, keratin 5, and mesothelin in lung carcinomas of different types: an immunohistochemical analysis of 596 tumors in comparison with epithelioid mesotheliomas of the pleura. Am J Surg Pathol 2003;27:150-158.

5 Roberts F, Harper CM, Downie I, et al. Immunohistochemical analysis still has a limited role in the diagnosis of malignant mesothelioma. A study of thirteen antibodies. Am J Clin Pathol 2001;116:253-262.

6 Abutaily AS, Addis BJ, WR Roche. Immunohistochemistry in the distinction between malignant mesothelioma and pulmonary adenocarcinoma: a critical evaluation of new antibodies. J Clin Pathol 2002; 55:662-668.

7 Ordóñez NG. The immunohistochemical diagnosis of mesothelioma: a comparative study of epithelioid mesothelioma and lung adenocarcinoma. Am J Surg Pathol 2003;27:1031-1051.

8 Marks A, Sutherland DR, Bailey D, et al. Characterization and distribution of an oncofetal antigen (M2A antigen) expressed on testicular germ cell tumors. Br J Cancer 1999;80:569-578.

9 Kahn HJ, Bailey D, Marks A. Monoclonal antibody D240, a new marker of lymphatic endothelium, reacts with Kaposi's and a subset of angiosarcomas. Mod Pathol 2002;15:434-440.

10 Fogt F, Pascha TL, Zhang PJ, et al. Proliferation of D240-expressing intestinal lymphatic vessels in the lamina propria in inflammatory bowel disease. Int J Mol Med 2004;13:211-214.

11 Fogt F, Zimmerman RL, Ross HM, et al. Identification of lymphatic vessels in malignant, adenomatous and normal colonic mucosa using the novel immunostain D2-40. Oncol Rep 2004;11:47-50.

12 Kahn HJ, Marks A. A new monoclonal antibody, D2-40, for detection of lymphatic invasion in primary tumors. Lab Invest 2002;82:1255-1257.

13 Battifora H, Mccaughey WTE. Tumors of the serosal membranes. In: Rosai J, Sobin LH (eds). Atlas of Tumor Pathology, Third Series. Armed Forces Institute of Pathology: Washington, DC, 1995, pp 11-88.

$14 \mathrm{Kim}$ MK, Kim S. Immunohistochemical profile of common epithelial neoplasms arising in the kidney. Appl Immunohistochem Mol Morphol 2002;10: 332-338.

15 Gulyás M, Dobra K, Hjerpe A. Expression of genes encoding for proteoglycans and Wilms' tumour susceptibility gene 1 (WT1) by variously differentiated benign human mesothelial cells. Differentiation 1999; 65:89-96.

16 Goldstein NS, Bassi D, Uzieblo A. WT1 is an integral component of an antibody panel to distinguish pancreaticobiliary and some ovarian epithelial neoplasms. Am J Clin Pathol 2001;116:246-252.

17 Goldstein NS, Uzieblo A. WT1 immunoreactivity in uterine papillary serous carcinomas is different from ovarian serous carcinomas. Am J Clin Pathol 2002; 117:541-545.

18 Lee BH, Hecht JL, Pinkus JL, et al. WT1, estrogen receptor, and progesterone receptor as markers for breast or ovarian primary sites in metastatic adenocarcinoma to body fluids. Am J Clin Pathol 2002;117: $745-750$.

19 Shimizu M, Toki T, Takagi Y, et al. Immunohistochemical detection of the Wilm's tumor gene (WT1) in epithelial ovarian tumors. Int J Gynecol Pathol 2000;19:158-163.

20 Acs G, Pasha T, Zhang PJ. WT1 is differentially expressed in serous, endometrioid, clear cell, and mucinous carcinomas of the peritoneum, fallopian tube, ovary, and endometrium. Int J Gynecol Pathol 2004;23:110-118.

21 Attanoos RL, Dojcinov SD, Webb R, et al. Antimesothelial markers in sarcomatoid mesothelioma and other spindle cell neoplasms. Histopathology 2000;37:224-231.

22 Lucas DR, Pass HI, Madan SK, et al. Sarcomatoid mesothelioma and its histological mimics: a comparative immunohistochemical study. Histopathology 2003;42:270-279. 